In Crescendo. Institucional; 2017 8(1): 30-40

Fecha de recepción: 20 de marzo de 2017

Fecha de aceptación: 30 de marzo de 2017

\title{
MetaAnÁlisis de unA INTERVENCIÓN EDUCATIVA PARA EL USO ADECUADO DE MEDICAMENTOS, 2014
}

\section{ANALYSES OF AN EDUCATIONAL INTERVENTION FOR PROPER USE OF DRUGS, 2014}

Liz Elva Zevallos Escobar*

\section{RESUMEN}

$E^{1}$ objetivo del presente estudio fue determinar el impacto de una intervención educativa para el uso adecuado de medicamentos en pobladores de los distritos de Chimbote y Nuevo Chimbote durante el año 2014. Se realizó una investigación de tipo longitudinal, preexperimental, con un nivel de investigación de enfoque cuantitativo. Se realizó una encuesta domiciliaria sobre el uso adecuado de medicamentos y se desarrolló una intervención educativa en 1923 pobladores, que consistió en una charla educativa, entrega de trípticos y visitas domiciliarias con la finalidad de mejorar sus conocimientos. A través de la prueba estadística de McNemar se determinó que la intervención educativa mostró un cambio favorable en el conocimiento del uso adecuado de medicamentos. Se concluye finalmente que la intervención educativa tiene un impacto positivo en el conocimiento del uso adecuado de medicamentos.

Palabras clave: intervención educativa, uso adecuado de medicamentos

\begin{abstract}
The aim of this study was to determine the impact of an educational intervention for proper use of drugs in residents of the districts of Chimbote and New Chimbote during 2014. longitudinal research, preexperimental type was conducted with a level of research quantitative approach. a household survey on the appropriate use of medicines was carried out and an educational intervention in 1923 settlers, consisting of an educational talk, delivering leaflets and home visits in order to improve their knowledge was developed. Through statistical McNemar test it was determined that the educational intervention showed a favorable change in the knowledge of the proper use of medications. It concludes that the educational intervention has a positive impact on the knowledge of the proper use of medications.
\end{abstract}

Keywords: appropriate use of medications, educational intervention

* Químico farmacéutico. Magíster en Farmacología. Docente investigadora de la Escuela Profesional de Farmacia y Bioquímica. Uladech Católica. Correo electrónico: liselvita@hotmail.com 


\section{INTRODUCCIÓN}

Consideramos al medicamento como toda sustancia y sus asociaciones o combinaciones, destinadas a prevenir, diagnosticar, aliviar o curar enfermedades en humanos y animales, con fines de controlar o modificar sus estados fisiológicos o fisiopatológicos. En este sentido todo medicamento, independientemente de su origen o naturaleza, debe garantizar tanto al individuo como a la sociedad su calidad, así como comprobar que su seguridad y eficacia contribuirá de manera importante a la solución de problemas que justifique su uso racional.(1) Todos los medicamentos pueden tener un riesgo asociado a su uso. Lógicamente, el perfil de efectos indeseados (en frecuencia y gravedad) es distinto para cada fármaco y depende de sus propiedades farmacodinámicas y farmacocinéticas. Si los fármacos no se utilizan adecuadamente, es más fácil que su relación beneficio/riesgo se vuelva desfavorable. No existe en la población el concepto de que el medicamento es una sustancia que entraña riesgos y que, por tanto, debería utilizarse con la orientación de un profesional capacitado para ello. $(2,3)$

Debido a que en los países menos desarrollados la condición humana todavía está marcada por el hambre, la enfermedad y la muerte precoz, la percepción de la salud que tienen sus poblaciones es muy diferente a la que existe en los países industrializados, por tanto la población común que toma medicamentos por lo general conoce poco sobre ellos y sobre la forma en que deben ser utilizados, pudiendo tener ideas o creencias equivocadas que posibilitan un mal empleo de los productos, lo que puede hacerlos menos efectivos y/o más peligrosos además de la promoción publicitaria algunas veces descontrolada y que incluso puede resultar engañosa.(2)

Hoy en día, el consumo de medicamentos es un fenómeno de creciente relevancia, que está siendo motivada por diversos factores que se asocian a valores que predominan en la sociedad actual. Entre estos factores es posible mencionar, el desconocimiento de la población en el tema, la deficiente cobertura en salud, mala calidad de servicios de salud, el aumento de suministros de medicamentos alternativos, la disponibilidad, la venta sin restricciones y propaganda de productos farmacéuticos en los medios de comunicación. (4) En el Perú, se asocian a estos factores, paradigmas y creencias sobre el uso de medicamentos que influyen directamente en la decisión final de una persona; generando de esta forma automedicación irresponsable, resistencia bacteriana, reacciones adversas a medicamentos, entre otras; lo cual crea un problema de salud pública que influye negativamente en la credibilidad de los sistemas de salud.(5)

La Organización Mundial de la Salud (OMS) calcula que más del 50 \% de todos los medicamentos se recetan, se dispensan o se venden en forma inadecuada, al mismo tiempo, alrededor de un tercio de la población mundial carece de acceso a medicamentos esenciales, y el 50 \% de los pacientes los toman de forma incorrecta. (4) Los consumidores necesitan información detallada acerca de los fármacos que les permita utilizar los fármacos con seguridad y efectividad. Para que la información sea útil se debe presentar en un formato que pueda ser comprendido fácilmente por los consumidores.(6) 
Ante el contexto antes planteado, se propuso como objetivo determinar el impacto de una intervención educativa para el uso adecuado de medicamentos en pobladores de Chimbote y Nuevo Chimbote, antes y después de la intervención educativa durante el año 2014.

\section{METODOLOGÍA}

Según Del valle (7) y Maldonado (2) modificada.

\section{Diseño de la investigación}

El presente trabajo de investigación corresponde a un estudio de tipo longitudinal, preexperimental, con un nivel de investigación de enfoque cuantitativo. A un grupo se le aplica una prueba previa al estímulo o tratamiento experimental, después se le administra el tratamiento y, finalmente, se le aplica una prueba posterior al tratamiento. Se utiliza un solo grupo de control y experimento.

El esquema a seguir es el siguiente:<smiles></smiles>

Donde:

$\mathrm{GE}=$ Grupo experimental

$\mathrm{O}=$ Pobladores

01 = Pretest aplicado al grupo experimental

02 = Postest aplicado al grupo experimental

$\mathrm{X}=$ Intervención educativa

\section{Población y muestra}

Población: El tamaño del universo se determinó teniendo como referente la información brindada por las postas médicas y dirigentes de los pueblos jóvenes, urbanizaciones y asentamientos humanos de Chimbote y Nuevo Chimbote: asentamiento humano Antenor Orrego, Chimbote; asentamiento humano Corazón de Jesús, Chimbote; urbanización Antúnez de Mayolo, Chimbote; pueblo joven Alto Perú, Chimbote; pueblo joven 3 de Octubre, Nuevo Chimbote; asentamiento humano 19 de Marzo de San Luis, Nuevo Chimbote; urbanización Nicolás Garatea 1. ${ }^{a}$ etapa, Nuevo Chimbote; pueblo joven Villa María, Nuevo Chimbote.

Muestra: Para la estimación del tamaño muestral(n), se aplicó la siguiente fórmula:

$$
\mathrm{n}=\frac{\mathrm{z} 2 * \mathrm{P}^{*} \mathrm{Q} * \mathrm{~N}}{\mathrm{~d} 2(\mathrm{~N}-1)+\mathrm{z} 2 * \mathrm{P} * \mathrm{Q}}
$$

$\mathrm{P}=$ Proporción: $50 \%$ de la población

$\mathrm{Q}=$ Probabilidad de fracaso: $1-\mathrm{P}$

d = Precisión: $5 \%$ (cuanto se aleja la muestra del verdadero porcentaje del universo) 
$z=1.96$. (Nivel de confianza 95\% para un nivel de significación del $5 \%$ )

$\mathrm{n}=$ tamaño de muestra

$\mathrm{N}=$ Universo

Una vez determinado el tamaño muestral en cada población, se tuvo en cuenta los criterios de inclusión y exclusión para la selección de los grupos intervenidos.

\section{Criterios de inclusión y exclusión}

\section{Criterios de inclusión}

- Pobladores que usaron medicamentos.

\section{Criterios de exclusión}

- Pobladores con enfermedades terminales

- Pobladores que no aceptaron a participar en el estudio

- Pobladores que no participaron en la encuesta final por razones de no encontrarse en su domicilio por motivos de trabajo, viaje a otro lugar, entre otros motivos.

Finalmente, con base a estos criterios de la muestra se seleccionó a 1923 pobladores captados en las comunidades de Chimbote y Nuevo Chimbote a quienes se realizó la intervención educativa.

\section{Técnicas e instrumentos de recolección de datos}

La presente investigación se desarrolló en dos etapas: la primera fue considerada una evaluación de preprueba que nos permitió identificar a los pobladores que usaron medicamentos y los conocimientos previos que tenían respecto a su uso adecuado.

La encuesta presentó 8 preguntas que corresponden al conocimiento de las indicaciones de medicamentos, frecuencia de administración, dosis, reacciones adversas, identificación de reacciones adversas, recomendación profesional, lugar de adquisición, discriminación entre genérico y comercial.

Se utilizó un consentimiento informado (Anexo I) donde el investigador notificó al entrevistado los objetivos del estudio y sus fines.

La intervención educativa se dio a través de una presentación adecuada a los pobladores a través de una charla educativa, así como también a través de material impreso del tríptico sobre temas específicos relacionados con el uso adecuado de medicamentos. El contenido de la ponencia y la presentación del material impreso tuvieron en cuenta lenguaje sencillo y claro así como mensajes cortos e ideas concretas. La evaluación posprueba se llevó a cabo luego de un mes de la intervención a través de un cuestionario validado para evaluar el conocimiento del uso adecuado. La información fue recabada y analizada mediante la utilización de un formato realizado en una hoja electrónica del programa Microsoft Office Excel 2010, para su valoración estadística. 


\section{Evaluación del impacto de la intervención educativa}

Criterios de aceptación: Según el cuestionario se deben responder 7 preguntas de 8 ( $87,5 \%$ de respuesta adecuada $=$ uso adecuado, menos de 87,5\% uso inadecuado). Con estas respuestas se realizó un análisis descriptivo de frecuencias (antes/después) de acuerdo a los criterios mencionados anteriormente que permitieron comprobar el avance de conocimientos de forma individual.

\section{Plan de análisis de datos}

Se realizó una tabla de frecuencias de acuerdo con la clasificación anterior, capaz de evidenciar de forma porcentual y por frecuencia la cantidad de conocimiento de los individuos y se comparó un conocimiento previo con el conocimiento analizado de la encuesta de seguimiento.

Se utilizó la prueba estadística de chi cuadrado de McNemar para determinar la efectividad de la intervención educativa impartida a la población en una tabla que compara antes de la charla y después de la misma con el valor significativo de $\mathrm{p}<0,05$ o altamente significativo $\mathrm{p}<0,01$.

\section{Consideraciones éticas}

Se solicitó el consentimiento informado de cada participante, de manera que se enteraron de lo que se trató el presente estudio, considerando y haciendo énfasis en la confidencialidad de la información y su identidad. Los datos que fueron recogidos en el curso del estudio se documentaron de manera anónima, la información recopilada sólo fue utilizada con fines de investigación.

\section{RESULTADOS}

Tabla 1. Patrones de uso de medicamentos en pobladores de Chimbote y Nuevo Chimbote, antes y después de la intervención educativa durante el año 2014

\begin{tabular}{|c|c|c|c|c|c|c|c|c|}
\hline \multirow{4}{*}{ CONOCIMIENTOS } & \multicolumn{8}{|c|}{$\begin{array}{l}\text { CONOCIMIENTO DEL USO ADECUADO DE } \\
\text { MEDICAMENTOS }\end{array}$} \\
\hline & \multicolumn{4}{|c|}{ ANTES } & \multicolumn{4}{|c|}{ DESPUÉS } \\
\hline & \multicolumn{2}{|c|}{ Sí } & \multicolumn{2}{|c|}{$\mathrm{NO}$} & \multicolumn{2}{|c|}{ Sí } & \multicolumn{2}{|c|}{$\mathrm{NO}$} \\
\hline & N. ${ }^{\circ}$ & $\%$ & N. ${ }^{\circ}$ & $\%$ & N. ${ }^{\circ}$ & $\%$ & N. ${ }^{\circ}$ & $\%$ \\
\hline Indicaciones & 1325 & 69 & 598 & 31 & 1583 & 82 & 340 & 18 \\
\hline Frecuencia & 1087 & 57 & 836 & 43 & 1365 & 71 & 558 & 29 \\
\hline Dosis & 1115 & 58 & 808 & 42 & 1448 & 75 & 475 & 25 \\
\hline Reacciones adversas & 476 & 25 & 1447 & 75 & 969 & 50 & 954 & 50 \\
\hline $\begin{array}{l}\text { Identificación de una reacción } \\
\text { adversa }\end{array}$ & 349 & 18 & 1574 & 82 & 692 & 36 & 1231 & 64 \\
\hline Por recomendación profesional & 1102 & 57 & 821 & 43 & 1529 & 80 & 394 & 20 \\
\hline Lugar de adquisición & 1615 & 84 & 308 & 16 & 1788 & 93 & 135 & 7 \\
\hline $\begin{array}{l}\text { Discriminación entre genérico y } \\
\text { comercial }\end{array}$ & 1031 & 54 & 892 & 46 & 1160 & 60 & 763 & 40 \\
\hline
\end{tabular}

Fuente: Cuestionario aplicado 
Gráfico 1. Patrones de uso de medicamentos en pobladores de Chimbote y Nuevo Chimbote, antes y después de la intervención educativa durante el año 2014

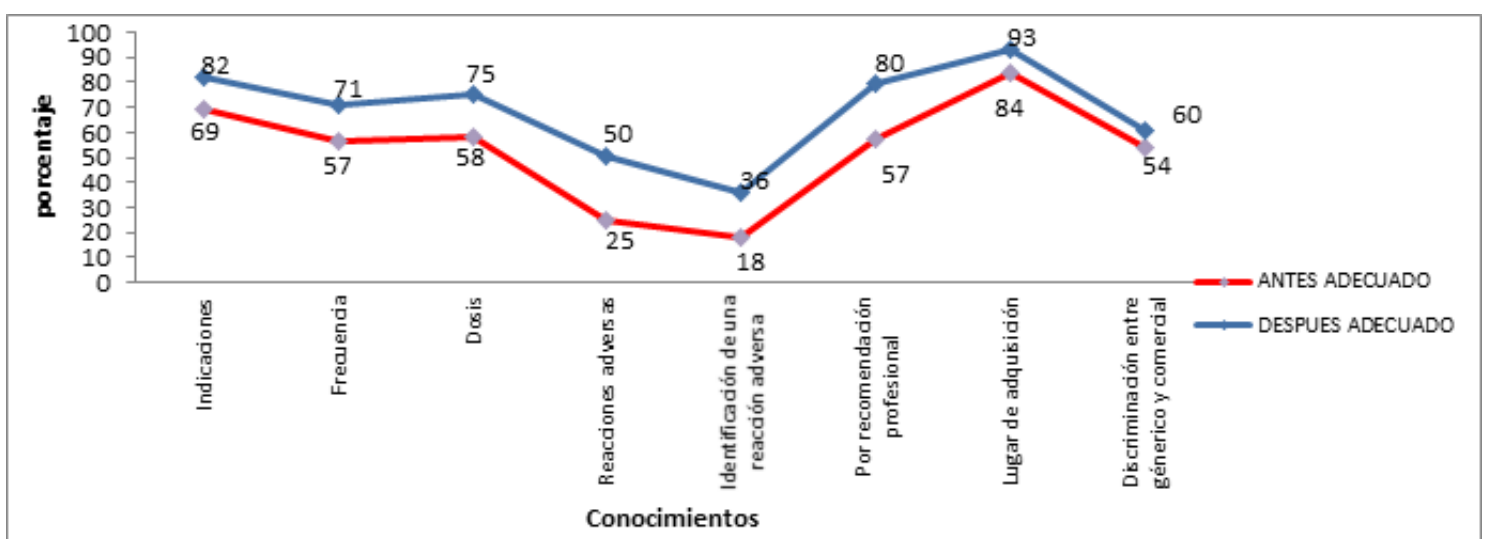

Fuente: Cuestionario aplicado.

\section{Interpretación}

En la tabla 1 y gráfico 1 se describe el nivel de conocimiento de los pobladores en relación a los patrones de uso de sus medicamentos. Se puede observar que antes de la intervención educativa las preguntas con menor porcentaje de respuestas correctas fueron respecto a las reacciones adversas ( $25 \%$ ) e identificación de las reacciones adversas (18\%), que luego de la intervención sólo mejoran en un $50 \%$ y 36\% de aciertos en sus respuestas. Por otra parte, la pregunta con mayor porcentaje de aciertos en los pobladores, antes de la intervención, fue el lugar de adquisición de sus medicamentos en establecimientos de salud con un $84 \%$ mejorando a un $93 \%$ después de la intervención. Se puede observar además que antes de la intervención más de la mitad de la población conoce sus indicaciones (69\%), frecuencia de administración (57\%) y dosis (58\%), que mejora considerablemente luego de la intervención educativa en un $82 \%, 71$ y $75 \%$, respectivamente.

Tabla 2. Uso adecuado de medicamentos en pobladores de Chimbote y Nuevo Chimbote, antes y después de la intervención educativa durante el año 2014

\begin{tabular}{|c|c|c|c|c|c|c|c|}
\hline \multirow{2}{*}{$\begin{array}{l}\text { Antes de la inter- } \\
\text { vención educativa }\end{array}$} & \multicolumn{3}{|c|}{ Después de la intervención educativa } & \multirow[b]{2}{*}{$\%$} & \multirow{2}{*}{ TOTAL } & \multirow{2}{*}{$\%$} & \multirow{2}{*}{ Significancia $(p)$} \\
\hline & $\begin{array}{l}\text { Uso adecuado de } \\
\text { medicamentos }\end{array}$ & $\%$ & $\begin{array}{l}\text { Uso inadecuado de } \\
\text { medicamentos }\end{array}$ & & & & \\
\hline $\begin{array}{l}\text { Uso adecuado de } \\
\text { medicamentos }\end{array}$ & 1025 & 53.3 & 351 & 18.3 & 1376 & 71.6 & \\
\hline $\begin{array}{l}\text { Uso inadecuado de } \\
\text { medicamentos }\end{array}$ & 0 & 0.0 & 547 & 28.4 & 547 & 28.4 & $0.0000^{*}$ \\
\hline TOTAL & 1025 & 53.3 & 898 & 46.7 & 1923 & 100.0 & \\
\hline
\end{tabular}

$\mathrm{p}<0,01$

Fuente: Cuestionario aplicado. 


\section{Interpretación}

La tabla 2 mostró una mejoría considerable del nivel de conocimientos después de la intervención educativa en los pobladores. La prueba de McNemar muestra un valor de $p<0,01$ que indica un valor muy significativo de mejora en el uso de medicamentos

\section{DISCUSIÓN}

La mayoría de las intervenciones educativas destinadas a mejorar el uso de medicamentos en la población común han sido poco documentadas. Los resultados obtenidos en la aplicación de la intervención educativa permiten considerar que fue eficaz para mejorar los conocimientos de pobladores y disminuir la frecuencia de su uso inadecuado en relación a creencias y prácticas erróneas, de manera que promueven su uso adecuado.

Para el estudio, la muestra en investigación estuvo representada por 1923 pobladores quienes fueron encuestadas acerca del de medicamentos, considerando que su uso está determinado por una complejidad de factores, entre los que se encuentran conocimientos y actitudes, así como factores socio-demográficos, culturales, económicos, políticos y regulatorios que conforman el contexto donde ocurre la demanda y oferta de medicamento (1).

Luego de la aplicación de la intervención educativa las frecuencias de respuestas aumentaron los aciertos en varios de los ítems evaluados, que demuestra la influencia en los pobladores, como podemos apreciar en la tabla 1 donde el $69 \%$ conocía las indicaciones para el uso de sus medicamentos, luego de la intervención educativa podemos ver que mejoró el nivel de conocimiento a $82 \%$, considerando una diferencia significativa de $13 \%$.

Cuando se analiza el cumplimiento del tratamiento farmacológico, las cifras de algunos estudios previos señalan que entre un tercio y la mitad de pacientes no toman sus medicamentos de la forma indicada, siendo uno de los problemas más significativos que enfrentamos en relación al uso de medicamentos (8).

El cumplimiento del tratamiento farmacológico implica también el conocimiento de la frecuencia de administración de sus medicamentos en cuanto a intervalos de administración y días de tratamiento que permiten el uso correcto del medicamento, la tabla muestra que el $57 \%$ de los pobladores respondieron que conocían la frecuencia de administración antes de la intervención, luego de la intervención mejoró a 71 \%, siendo así que el $14 \%$ de los pobladores mejoraron sus conocimientos con la acción formativa.

Con respecto al conocimiento de la dosis antes de la intervención el $58 \%$ de los pobladores conocían su uso adecuado, se destaca que la falta de información que tienen los consumidores, acceso sin restricciones a los medicamentos, dosis y duración inadecuadas, constituyen elementos de irracionalidad, es decir que tomar dosis inadecuadas o de forma irregular, puede repercutir en eventos que causen daño al consumidor o puede que no se observe el efecto esperado, luego de la intervención educativa se observa que 
el $75 \%$ de los pobladores entendieron la importancia de conocer las dosis de cada medicamento que usan (9).

Los resultados en la tabla 01 antes de la intervención educativa muestran un $25 \%$ y $18 \%$ de conocimientos de los pobladores referente a las reacciones adversas y su identificación, respectivamente, lo que demuestra que desconocen los riegos que asumen al consumir sus medicamentos, estos datos demuestran la importancia de educar a los pobladores respecto a que ningún medicamento es inocuo y sus efectos adversos pueden llegar a ser catastróficos si se utiliza de manera irracional. Se rescata que después de la intervención el 50\% y 36\% conocen e identifican las reacciones adversas de los medicamentos que usaron, si bien es cierto se ve una mejora notable estos resultados indican que debemos incidir en reforzar los conocimientos básicos en relación a las reacciones adversas medicamentosas, a fin de que puedan detectarlas y notificarlas a los profesionales responsables (1).

Como se puede observar en el gráfico 1 el $57 \%$ de los pobladores usaban el medicamento por recomendación de un profesional antes de la intervención educativa,

mientras que un $80 \%$ lo uso por recomendación de un profesional después de la intervención existiendo una mejora considerable en los aciertos de un $23 \%$, consideramos que es responsabilidad del paciente seguir el tratamiento prescrito por el médico o seguir los consejos del profesional químico farmacéutico, es decir, corresponde al paciente usar de forma racional el medicamento, por ello importancia de educarlos en el uso correcto de estos.

En cuanto a donde adquieren sus medicamentos antes de la intervención el $84 \%$ indicó que lo hacían en lugares adecuados, luego de la intervención mejoró al 93 \% de los pobladores que saben dónde adquirir sus medicamentos correctamente. En el Perú la facilidad de tener acceso a los medicamentos sin necesidad de una receta médica es muy frecuente en las farmacias y boticas, si bien es cierto existen medicamentos OTC de venta sin prescripción, también están los antibióticos de los cuales los usuarios hacen uso indiscriminado e inadecuado (10).

Se percibió entre los pobladores encuestados cierta desconfianza respecto al uso de medicamentos genéricos, muchos de ellos adquiridos en establecimientos del Ministerio de Salud (Minsa) y el seguro social (EsSalud), por eso el gráfico 1 muestra que el $54 \%$ de los pobladores se inclinaban por el uso de medicamentos genéricos. El gasto en medicamentos actualmente es un componente de constante crecimiento de costos en relación al cuidado de la salud, que representan un motivo de preocupación por el desvío de los fondos de las economías familiares a la compra de productos de poco valor terapéutico como combinaciones inadecuadas, riesgosas e irracionales, básicamente por el desconocimiento de los consumidores. El $60 \%$ de los pobladores capacitados luego de la intervención educativa indicaron su preferencia por los medicamentos genéricos considerando que el valor y eficacia terapéutica es similar a los medicamentos de marca, lógicamente teniendo en cuenta la procedencia de los genéricos y el lugar de adquisición (11). 
A pesar de la corta duración de las intervenciones realizadas, los resultados han sido favorables, como podemos observar en la tabla 2 antes de la intervención educativa el $53,3 \%$ de los pobladores que participaron indicaron el conocimiento del uso adecuado de medicamentos, lo que significa que contestaron más de 7 respuestas correctas del cuestionario aplicado. Luego de la actividad educativa, se observó que del grupo intervenido el 18,3\% mejoraron significativamente los conocimientos sobre el uso correcto de sus medicamentos.

Luego de la intervención educativa, de manera global, la tabla 2 muestra que el 71,6\% de los pobladores conocen el uso adecuado de sus medicamentos, sin embargo, todavía existe un $28,4 \%$ de pobladores que aún no tienen el conocimiento suficiente del uso correcto de los medicamentos que usaron.

Es importante promover el uso adecuado de los medicamentos a través de sesiones educativas ya que su uso está determinado por aspectos conductuales, actitudinales, creencias y conocimientos adquiridos producto de la interacción del individuo en su entorno (1).

De los resultados en la tabla 2 , se determina un valor de $\mathrm{p}=0.0000$ que se considera un valor muy significativo; siendo este valor menor que 0,01 ( $p<0,01)$, por tanto se evidencia que existe diferencia significativa entre el nivel de conocimiento antes y después de la intervención educativa, por lo que nuestros resultados tiene un impacto positivo.

La prueba de McNemar se utiliza para decidir si hubo o no un cambio significativo entre una evaluación inicial y una final. Predice si existe un cambio en la respuesta de los elementos sometidos al estudio, y es aplicable a los diseños del tipo "antes-después" en los que cada elemento actúa como su propio control, que' permiten comprobar si hay diferencias entre frecuencias y proporciones, por lo que hace referencia a un conjunto de características que se consideran relevantes.

Diversos estudios sobre intervenciones educativas demuestran ser efectivos para ayudar o mejorar el uso adecuado de los medicamentos, medidos a través de la prueba estadística según McNemar, tal como lo evidencia Maldonado (2), utilizó estrategias de charlas educativas a estudiantes y midió la intervención educativa a corto y largo plazo mostrando una efectividad de la intervención educativa con un valor de $\mathrm{p}=0.001$.

Del Valle (7) en su estudio de educación sanitaria sobre el correcto uso de antiinflamatorios no esteroideos (AINEs) en padres de familia, los resultados muestran una diferencia significativa $(\mathrm{p}<0.0001)$, con lo que se comprobó en el estudio que de acuerdo a los conocimientos obtenidos por los padres de familia en estudio aumento el nivel de conocimiento.

Otro estudio realizado en España en el 2016, revela que la educación en el proceso del uso adecuado del medicamento, en pobladores que concurre a una farmacia comunitaria resultó significativa $(\mathrm{p}<0,001)$ en relación al nivel de sus conocimientos, también consideraron la prueba de McNemar (12).

No hay dudas de la importancia que tiene para la población la información y el conocimiento sobre los medicamentos que consume, pero es importante que sea un profesional 
capacitado quien brinda la información correcta y que dicha información no debe ser dada por publicidad u otras personas que no están debidamente preparadas, apoyándonos en la definición de uso racional de medicamentos ofrecida por la OMS. Es imprescindible saber de la medicación adecuada, la dosis y duración de tratamiento $(8,9)$.

\section{CONCLUSIONES}

1. La intervención educativa dirigida a los pobladores de los distritos de Chimbote y Nuevo Chimbote tuvo un impacto positivo, al mejorar su nivel de conocimiento respecto del uso adecuado de medicamentos.

2. La aplicación de una intervención educativa en los pobladores se relaciona con el aumento del nivel de conocimientos respecto a los patrones de uso de medicamentos.

3. Existió un cambio significativo entre el conocimiento previo a la intervención educativa y posterior a ésta, considerando que mejoró el conocimiento de los pobladores respecto al uso adecuado de los medicamentos.

\section{REFERENCIAS BIBLIOGRÁFICAS}

1. Ávila A. Determinantes relacionados con la tenencia de medicamentos y su uso racional. [Tesis doctoral]. Universidad Autónoma de Barcelona. Bellaterra. 2011. [Consultado en marzo 2016] Disponible en: http://www.tdx.cat/bitstream/handle/10803/48519/aaldel.pdf?sequence $=1$

2. Maldonado J. Efectos a corto y largo plazo de una intervención educativa sobre uso adecuado de medicamentos en estudiantes de colegios fiscales de la ciudad de Quito. sis doctoral]. Universidad Autónoma de Barcelona. Barcelona. 2004. [Consultado en marzo 2016] Disponible en: https://www.icf.uab.es/universidad/treballs/JCMaldonado.pdf

3. Heineck I, Schenkel E. y Vidal J. Medicamentos de venta libre en el Brasil. Rev Panam Salud Publica/Pan Am J Public Health. 1998; 3(6): 385-391. [Consultado en febrero 2016] Disponible en: http://www.scielosp.org/pdf/rpsp/v3n6/3n6a4.pdf

4. Moya S. Estudio-prevalencia de Automedicación en Consultantes a un Centro de Atención ambulatorio adosado a un hospital de una Comuna de Santiago. [Tesis]. Universidad de Chile. 2012 [Consultado en febrero 2016]. Disponible en: http:// repositorio.uchile.cl/bitstream/handle/2250/111220/moya sa.pdf?sequence $=1$

5. Dirección General de Medicamento Insumos y Drogas-DIGEMID. Manual para la enseñanza del uso racional de medicamentos a la comunidad. OPS.2005. [Consultado en febrero 2016] Accesible desde: http://www.digemid.minsa.gob.pe/UpLoad \%5CUpLoaded \%5CPDF/1 al 12.pdf

6. Ciciriello S, Johnston RV, Osborne RH, Wicks I, deKroo T, Clerehan R, et al. Intervenciones educativas multimedia para los pacientes sobre medicamentos prescritos y de venta libre. Rev. Cochrane. [Consultado en julio 2016]. Accesible desde: http:// 
www.cochrane.org/CD008416/COMMUN multimedia-programs-for-educating-patients-about-medications

7. Del Valle L. Educación Sanitaria sobre el correcto uso de antiinflamatorios no esteroideos (AINEs) utilizados para tratar el dolor y la fiebre, dirigida a padres de familia de niños de 1-6 años que asisten a colegios ubicados en Ciudad San Cristóbal zona 8 de Mixco. [Tesis Doctoral]. Universidad de San Carlos de Guatemala. Guatemala. 2012. [Citado abril del 2015]. Disponible en: http://biblioteca.usac.edu.gt/ tesis/06/06 3363.pdf

8. García A. Caracterización epidemiológica del consumo de medicamentos por la población adulta de Cuba. 2007- 2010. [Tesis Doctoral]. Escuela Nacional Pública. Cuba. 2011. [Citado junio del 2016]. Disponible en: http://files.sld.cu/revsalud/files/2013/03/tesisanajuliagarcia.pdf

9. Baes C. El uso racional del medicamento fundamento de la intervención administrativa en el sector farmacéutico. [Tesis Doctoral]. Universidad de Granada. España. 2010. Disponible en: http://www.ugr.es/ sej03266/actividad/red medicamentos/repositorio/TESIS/TESIS CELINE.pdf

10. Ramos J. Caracterización de la práctica de automedicación en la población residente del distrito de ate de la provincia de Lima, marzo 2014. [Tesis]. Universidad Nacional Mayor de San Marcos. Perú. 2014. [Citado julio del 2016]. Disponible en: http:// cybertesis.unmsm.edu.pe/bitstream/cybertesis/3647/1/Ramos rj.pdf

11. Vivar D. Consumo y valor farmacoterapéutico de los 100 medicamentos más vendidos sin receta médica en farmacias y boticas del Perú, registrados en el IMS 19971998. [Tesis]. Universidad Nacional Mayor de San Marcos. Perú. 2002. Disponible en: http://sisbib.unmsm.edu.pe/bibvirtualdata/tesis/salud/vivar t d/t completo.pdf

12. Huarte J. Impacto de la educación en el proceso del uso adecuado del medicamento en cuidadores formales de pacientes habituales de una farmacia comunitaria de Pamplona. Farmacéuticos Comunitarios. 2016;8(1). Disponible en: http:// farmaceuticoscomunitarios.org/sites/default/files/suplemento/pdf/205.pdf 